Revisión bibliográfica

Volumen 31(3):803-819. Septiembre-diciembre, 2020

e-ISSN 2215-3608, doi:10.15517/am.v31i3.40217

http://www.revistas.ucr.ac.cr/index.php/agromeso

\title{
Alimentación: factor estratégico durante la crianza artificial de terneros provenientes de lecherías ${ }^{1}$
}

\section{Feeding: a strategic factor for artificial rearing of calves from dairy farms}

\author{
Ana María Nemocón-Cobos ${ }^{2}$, Joaquín Angulo-Arizala², Jorge Alberto Gallo-Marín ${ }^{2}$, Liliana Mahecha-Ledesma
}

1 Recepción: 7 de enero, 2020. Aceptación: 19 de mayo, 2020. Esta revisión formó parte del trabajo de Maestría en Ciencias Animales de la primera autora "Caracterización y evaluación bio-económica la cría de terneros provenientes de lechería especializada, con destino futuro a la producción de carne en trópico alto". Universidad de Antioquia, Facultad de Ciencias Agrarias. Medellín, Colombia.

2 Universidad de Antioquia, Facultad de Ciencias Agrarias, Grupo de Investigación en Ciencias Agrarias (GRICA). AA1126, Ciudadela Robledo, Cra 75 No 65-87. Medellín, Colombia. Teléfono +572199148. ana.nemocon@udea.edu.co (https://orcid.org/0000-0003-43006843); joaquin.angulo@udea.edu.co (autor para correspondencia, https://orcid.org/0000-0003-3352-8795); jalberto.gallo@udea.edu.co (https://orcid.org/0000-0002-1891-9141); liliana.mahecha@udea.edu.co (https://orcid.org/0000-0003-3377-8399).

\section{Resumen}

Introducción. Los sistemas de lechería especializada en Colombia, representan el sector más tecnificado de la producción bovina, pero presentan retos en torno a la rentabilidad y competitividad con el mercado de productos lácteos importados, que establece una desventaja para la producción local. Sin embargo, la crianza de machos y hembras de descarte, para producción de carne, puede ser una alternativa competitiva. Objetivo. Profundizar en el manejo de la alimentación durante la crianza de terneros provenientes de lecherías. Desarrollo. La crianza artificial consiste en el manejo, cuidado y alimentación de los terneros hasta el destete y su objetivo es lograr que los animales dependan menos nutricionalmente de la leche, a la menor edad posible, y puedan incorporar alimentos sólidos a la dieta. La a alimentación base de los terneros durante la crianza es: calostro, leche o lactoreemplazador, alimento concentrado, y forraje. Se debe asegurar la medición de la calidad de calostro y ofrecerlo en mayor proporción durante la primer hora posparto. La leche o lactoreemplazador se deberá dar máximo durante ocho semanas con tendencia a disminuir el periodo sin afectar el desarrollo de los terneros, ofreciendo un producto de calidad composicional y sanitaria. El concentrado y la fuente de forraje, podrá ser ofrecida desde las primeras semanas de vida, garantizando la calidad y retirando los sobrantes para evitar problemas gastrointestinales. Conclusión. La cría como etapa crítica requiere un manejo estratégico de la alimentación. El suministro de calostro de calidad en el momento indicado, es la base de toda la etapa de producción. La alimentación líquida es esencial para la adaptación fisiológica y enzimática del tracto gastrointestinal, mientras que el alimento sólido es fundamental para el desarrollo ruminal.

Palabras clave: desarrollo ruminal, ganadería de leche, producción sostenible, rentabilidad, terneros.

\begin{abstract}
Introduction. The specialized dairy systems in Colombia represent the most technified sector of the cattle production, nevertheless it has challenges with profitability and competitiveness with the market of imported dairy products, which establishes a disadvantage for local production. However, the rearing of discarded calves for meat
\end{abstract}


production, can be a competitive alternative. Objective. To deepen the management of feeding in the calves rearing stage from dairy farms. Development. Artificial breeding includes the management, care, and feeding of calves until weaning and its objective is to reduce the nutritionally dependent of milk, at the lowest age possible, and to be able to incorporate solid foods into the diet. The basic diet of the calves during rearing includes the colostrum, milk or milk replacer, concentrated feed, and forage. It must be ensured colostrum quality measurement and the offer of quality colostrum, in the first hours postpartum. Milk or milk replacer should be supplied for eight weeks maximum with a tendency to decrease the period without affecting the development of the calves, offering a product of compositional and sanitary quality. The concentrate feed and the forage source can be offered from the first weeks of birth, guaranteeing quality of the diet and removing the feed scraps to avoid gastrointestinal problems. Conclusion. Rearing as a critical stage requires strategic feeding management. The supply of quality colostrum at the right time is the basis of the entire production stage. Liquid diet is essential for the physiological and enzymatic adaptation of the gastrointestinal tract, while solid diet is essential for ruminal development.

Keywords: ruminal development, dairy cattle, sustainable production, profitability, calves.

\section{Introducción}

En Colombia, los machos de lechería especializada se consideran un descarte y en la mayoría de veces, no representa un beneficio económico para el sistema productivo, porque el costo del transporte para enviarlos a sacrificio puede ser igual o superior al pago recibido por el animal. En el caso de las hembras de descarte, aunque estas tienen un precio de venta mayor que los machos, sería conveniente darle un destino diferente al de producción de leche, ya que al ser descartadas, no cumplen los parámetros productivos ideales y sin embargo, pasan a ser reemplazos en otros hatos, disminuyendo los estándares de calidad necesarios para el país (Gómez et al., 2017).

La utilización de machos y de hembras de descarte de las lecherías especializadas, para producción de carne, se convierte en una alternativa para diversificar el sector lechero. La producción cárnica con estos animales, se basa en tres etapas: a) crianza artificial, hasta los 60 días de edad, con pesos entre 70 y $75 \mathrm{~kg}$; b) recría, que va hasta que el animal alcance un peso entre 180 y $200 \mathrm{~kg}$, aproximadamente a los 210 días de edad; y c) finalización, donde los animales son llevados en promedio, hasta un peso de 330 a $350 \mathrm{~kg}$, a los 310 días de vida (Gonsolin, 2013).

El tipo y calidad de la alimentación determinan el tiempo que transcurra para cada etapa y, por ende, la rentabilidad y viabilidad económica del sistema productivo. Por lo tanto, una buena finalización de la crianza de machos y hembras de descarte, provenientes de lecherías, implica una buena ganancia de peso durante todas las etapas productivas. Esto puede ser posible con un buen manejo de las razas predominantes, entre ellas la Holstein, sobre la que se reporta un buen potencial para ganar peso, con una deposición tardía de grasa que se observa desde la etapa de cría, y con una baja proporción de grasa con relación a músculo en el tejido depositado, favoreciendo la producción de carne magra (Gonsolin, 2013).

En Estados Unidos, la principal forma de comercialización de la carne de ternero es bajo el sello "Natural", lo cual según el Departamento de Agricultura de Estados Unidos (USDA), hace referencia a los productos que no contienen ningún tipo de aditivo, colorante o saborizante artificial, químico o sintético, es decir, alimentos frescos sin procesar, identificados con un sello que así lo acredite. Para el 2008, Estados Unidos reportó un consumo per cápita de $0,3 \mathrm{~kg}$ de carne de ternero, valores significativos si se tiene en cuenta que la carne de ternero está asociada a la cocina internacional (USDA, 2013).

En Chile, la crianza tiene como objetivo la producción de "terneros lechales", los cuales, bajo un sistema de alimentación basado en leche y pasturas, alcanzan los $250 \mathrm{~kg}$, como peso ideal de faenado a una edad promedio de 
ocho meses, produce cortes de calidad, donde alrededor del 40 a $50 \%$ se distribuyen para venta directa en hoteles y restaurantes gourmet, y el porcentaje restante es para exportación, a países como Rusia (Ministerio de Agricultura y Fundación para la Innovación Agraria, 2010).

La alternativa de producción de carne con machos y hembras de descarte provenientes de lecherías especializadas, presenta como limitante para su implementación, el desconocimiento del manejo de puntos críticos durante la crianza de los animales, sobre todo cuando provienen de otras lecherías especializadas, siendo el principal factor crítico, el manejo de la alimentación, lo que podría incrementar la mortalidad, superando los límites máximos de referencia en pre-destete $(8,3-10 \%)$ y post-destete $(9,1 \%)$ (USDA, 2007; Elizondo-Salazar y Solís-Chaves, 2018).

El objetivo de esta revisión fue profundizar en el manejo de la alimentación, durante la crianza de terneros provenientes de lecherías.

\section{Crianza artificial}

La crianza artificial consiste en el manejo, cuidado y alimentación de los terneros hasta el destete, y su objetivo es lograr que los animales dependan menos de la leche para obtener sus nutrientes, a la menor edad posible, y puedan incorporar otros alimentos sólidos a la dieta (Dichio et al., 2015). En las terneras de reemplazo provenientes de lecherías, el periodo de crianza en promedio es de ocho semanas (Amado et al., 2019; Elizondo-Salazar y Monge-Rojas, 2019), mientras que en los machos se reporta un rango entre 3 y 8 semanas (Kehoe et al., 2007; Gonsolin, 2013; Eckert et al., 2015; Cheema et al., 2016; Daneshvar et al., 2017).

Los alimentos base en la dieta de los terneros durante la crianza son: calostro, leche o lactoreemplazador, alimento concentrado y forraje, dando siempre agua de buena calidad, a voluntad.

\section{Calostro}

La primera secreción posparto de la glándula mamaria es denominada calostro, y a través de esta, se provee al neonato de inmunidad pasiva (inmunoglobulinas), carbohidratos, grasa y proteína, esenciales para el metabolismo inicial (Morrill et al., 2012). Una adecuada inmunidad transferida, ha sido relacionada con menor mortalidad y mejor desarrollo de los animales (Dunn et al., 2017; Silva-Del-Río et al., 2017), y es necesaria, debido a que, durante la gestación, el tipo de placenta de los bovinos (epiteliocorial) impide el paso de inmunoglobulinas de la madre al feto; a eso se le suma, la incapacidad del sistema inmune para producir anticuerpos en las primeras semanas de vida (Conneely et al., 2013).

La cantidad y calidad del calostro es una parte crítica de la cría de terneros, por la necesidad de administrar cantidades adecuadas de inmunoglobulinas, citoquinas y factores de crecimiento que permitan un buen desarrollo del neonato (McGuirk y Collins, 2004). De igual forma, dicho suministro debe darse en las primeras 24 h del día, ya que, posterior a este lapso de tiempo, cambia la composición, pasando a ser del día dos al cinco, leche de transición, y de ahí en adelante, leche convencional (Espada et al., 2011; Salazar-Acosta y Elizondo-Salazar, 2019).

El suministro de calostro, no solo tiene importancia por la transferencia de inmunidad pasiva, sino que contiene altas cantidades de nutrientes y factores biológicamente activos, que estimulan la maduración y función neonatal del tracto gastrointestinal (e.g. factores de crecimiento, hormonas, citoquinas, antimicrobianos). La energía y la lactosa contenida en el calostro son críticas para la termogénesis y la termorregulación. Igualmente, vitaminas y minerales como calcio, magnesio, zinc, vitamina A, vitamina E, caroteno, riboflavina, vitamina B12, ácido fólico, colina y selenio, también se hallan en concentraciones significativas en el calostro de los bovinos (Godden et al., 2019). Por lo tanto, el suministro de una adecuada cantidad de calostro de buena calidad, determina el desarrollo digestivo y productivo de los terneros. 
Para lograr los niveles adecuados de absorción de calostro, y por ende, concentraciones adecuadas de inmunoglobulinas en el torrente sanguíneo del ternero, $10-14 \mathrm{mg} \mathrm{dl}^{-1} 24 \mathrm{~h}$ después del nacimiento (McCracken et al., 2017), es indispensable tener en cuenta el momento en el que se inicia el suministro de calostro, debido a la relación inversamente proporcional entre el tiempo que transcurre entre el nacimiento y el inicio del suministro de calostro, con la absorción intestinal. Por lo tanto, el suministro de calostro debería ser realizado entre las 0 y 2 horas de vida, teniendo en cuenta que la cantidad dependerá de la concentración de inmunoglobulinas (Dunn et al., 2017; Godden et al., 2019).

El suministro de una mayor cantidad de calostro durante la primera hora posparto, también es recomendable para tener una mayor protección en la salud de los terneros (Angulo et al, 2015), esto debido a que después de la primera hora empieza a disminuir el contenido de proteína del calostro y alcanza una reducción del $17 \%$ a las 5-6 h, aspecto que ha sido relacionado por otros autores, con la reducción de caseínas y específicamente con la reducción de glicopéptidos asociados a la caseína, los cuales tienen acción anti-rotavirus (Sobczuk-Szul et al., 2013; Inagaki et al. 2014).

Otros factores a tener en cuenta respecto a las características composicionales del calostro y la producción del mismo, en relación con la concentración de inmunoglobulinas, son la raza, la edad de la vaca, el estado nutricional pre-parto, y la duración del periodo seco. Respecto a la raza, se ha relacionado que podría haber un efecto de la genética con relación a la concentración de inmunoglobulinas, lo mismo que un efecto dilucional, es decir, que algunas razas podrían tener un mayor volumen, y una menor concentración de estas (Godden et al., 2019).

Con relación a la edad de la vaca, la concentración de inmunoglobulinas se ha asociado con el número de partos de la vaca (Shivley et al., 2018), reportándose una mayor concentración de inmunoglobulinas en vacas de tercer y cuarto parto $\left(107,4\right.$ a $\left.113,3 \mathrm{mg} \mathrm{ml}^{-1}\right)$, comparado con vacas de primer y segundo parto $\left(83,5\right.$ a $\left.92,9 \mathrm{mg} \mathrm{ml}^{-1}\right)$. Dicha tendencia se ha asociado a que las vacas adultas presentan un grado de desarrollo inmunitario completo (Kehoe, 2011).

En cuanto al estado nutricional de la vaca en el periodo pre-parto, no se ha hallado relación entre las restricciones nutricionales y algún efecto negativo sobre el calostro, puesto que, en dietas que no excedían los requerimientos energéticos de las vacas, se hallaron mayores concentraciones de inmunoglobulinas que en dietas con exceso de energía y no se encontraron diferencias significativas en la producción de calostro entre ambos casos (Mann et al, 2016). En lo que respecta a la duración del periodo seco y el volumen producido en la primera lactancia, se reporta que entre más corto sea el periodo seco, más baja puede ser la producción de calostro (Gavin et al., 2018).

La concentración de inmunoglobulinas es considerada el factor que se usa para determinar la calidad del calostro. Sin embargo, deberían también considerarse otros componentes como la grasa, la proteína y los minerales, que hacen un aporte importante a nivel nutricional (Zarei et al., 2017). Una buena composición nutricional, se verá reflejada en animales con mejor desarrollo en términos de crecimiento y producción (Shivley et al., 2018). La literatura reporta (Cuadro 1) diversos valores para solidos totales, grasa, proteína y lactosa, lo cual está asociado con el contexto productivo en el que se desarrolló cada uno de los estudios, en aspectos como el momento de toma de la muestra, la raza, el número de partos y el método de análisis.

En el manejo del suministro apropiado de calostro para las crías, es fundamental conocer el tipo y papel de las inmunoglobulinas, y saber identificar su concentración (Gelsinger et al., 2015).

Las inmunoglobulinas pueden ser de tres tipos IgG, IgA e IgM. Las más importantes cuando de inmunidad pasiva se trata son las IgG (Gelsinger et al., 2015) y representan entre 85 y $90 \%$ de las inmunoglobulinas totales (Conneely et al., 2013). Por lo tanto, la calidad del calostro, es determinada comúnmente según la concentración de IgG (Morrill et al., 2012). Estas inmunoglobulinas son responsables de activar las funciones bacteriolíticas, y aumentar el reconocimiento de patógenos y de fagocitosis de las bacterias por los leucocitos (Silva-Del-Río et al., 2017), y por lo tanto, de afrontar los retos inmunológicos y sanitarios del ternero. 
Cuadro 1. Valores promedio reportados para la composición nutricional del calostro (\%). Colombia, 2019.

Table 1. Average values reported for nutritional composition of colostrum (\%). Colombia, 2019.

\begin{tabular}{lcccc}
\hline Autor & Sólidos totales (\%) & Grasa (\%) & Proteína (\%) & Lactosa (\%) \\
\hline dos-Santos et al. (2017) & $22,6 \pm 1,35$ & $5,2 \pm 0,90$ & $15,1 \pm 1,77$ & NR \\
Zarei et al. (2017) & 27,2 & 4,6 & 18,5 & 2 \\
Thu-Hang et al. (2017) & $\mathrm{NR}$ & 4,8 & 21,4 & $\mathrm{NR}$ \\
Mann et al. (2016) & 26,52 & 5,01 & $\mathrm{NR}$ & $\mathrm{NR}$ \\
Morrill et al. (2012 & $\mathrm{NR}$ & 5,9 & 13,6 & 3 \\
Angulo et al. (2015) & 24,1 & 5,9 & - & $\mathrm{NR}$ \\
Salazar-Acosta y Elizondo-Salazar (2019) & $23,2 \pm 0,3$ & $5,7 \pm 0,1$ & $13,0 \pm 0,1$ & $3,1 \pm 0,1$ \\
\hline
\end{tabular}

NR: no reporta / NR: no reported

Las IgA e IgM, representan entre el 5 y $7 \%$ de las inmunoglobulinas totales, respectivamente, las primeras son responsables de proteger la superficie de las mucosas, y las segundas se encargan de ser la primera barrera de defensa en casos de infección generalizada (septicemia) (Godden et al., 2019).

La determinación e interpretación apropiada de la concentración de $\mathrm{IgG}$ en el calostro es importante porque esto define la calidad y determina la cantidad a suministrar (Gelsinger et al., 2015). A mayor concentración de IgG en el calostro, será considerado de mejor calidad, de allí la importancia de realizar evaluaciones para determinar su concentración. El valor mínimo recomendado en el calostro es de $50 \mathrm{~g} \mathrm{l}^{-1} \mathrm{de} \operatorname{IgG}$ o un valor igual o superior a 22 ${ }^{\circ}$ Brix, lo que implicaría un suministro de 41 de calostro por animal (Bielmann et al., 2010).

La concentración de IgG en el calostro, dependerá de la hora de evaluación, y durante la primera hora posparto, el momento en que se halla una mayor concentración. Posterior a ello, empezará a disminuir gradualmente hasta que pasan las primeras $24 \mathrm{~h}$ posparto (Godden et al., 2019). En un estudio se encontró una concentración de inmunoglobulinas a la hora 1, entre las 2 y $4 \mathrm{~h}$, y entre las 5 y $6 \mathrm{~h} \mathrm{de} \mathrm{72,75,72,98} \mathrm{y} 62,08 \mathrm{mg} \mathrm{ml}^{-1}$, respectivamente, mostrando una reducción significativa después de las 4 h (Angulo et al., 2015).

Debido a que la IgG es la que determina la calidad del calostro, es necesario realizar su medición. Para ello, se han utilizado técnicas como, el uso de calostrómetro (Bartier et al., 2015; Angulo et al., 2015) y la refractometría (Bielmann et al., 2010), que son consideradas de tipo indirecto y pueden realizarse en campo; mientras que, existen otras técnicas de tipo directo, que se realizan en laboratorio, como la inmunodifusión radial (RID) (Gelsinger et al., 2015), ELISA (Dunn et al., 2017), la espectroscopía de transmisión infrarroja (IRS) (Elsohaby et al., 2018) y recientemente, nuevos ensayos enzimáticos (Drikic et al., 2018) (Cuadro 2). Siendo estas últimas, las técnicas menos comunes y más recientemente exploradas.

La RID es una técnica de inmunodifusión cuantitativa, utilizada para determinar la concentración de un antígeno en un fluido biológico (Pechova et al., 2019). Este es un método que mide directamente IgG y se ha usado para desarrollar otros modelos que estiman la concentración de IgG, correlacionando la concentración de $\operatorname{IgG}$ con la gravedad específica, el índice de refracción o la proteína total plasmática (McCracken et al., 2017; Pechova et al., 2019). En cuanto al ELISA, esta técnica también es conocida como ensayo enzimático de inmunoabsorbancia, es considerada como una técnica de diagnóstico clínico "gold standard", para determinación y cuantificación de biomarcadores de proteínas (Thiha y Ibrahim, 2015).

La exactitud de la RID y ELISA fue evaluada y comparada, y se obtuvo una baja correlación entre los resultados de ambas técnicas para mediciones en calostro y plasma sin tratamiento térmico; adicionalmente se encontró que 
Cuadro 2. Valores promedio de la concentración de inmunoglobulinas G (IgG), reportada para distintas técnicas de evaluación. Colombia, 2019.

Table 2. Average values of immunoglobulin G (IgG) concentration, reported for different evaluation techniques. Colombia, 2019.

\begin{tabular}{|c|c|c|}
\hline Autor & Técnica de medición & IgG \\
\hline Gelsinger et al. (2015) & $\operatorname{RID}\left(\mathrm{mg} \mathrm{ml}^{-1}\right)$ & 77,94 \\
\hline Gelsinger et al. (2015) & $\operatorname{ELISA}\left(\mathrm{mg} \mathrm{ml}^{-1}\right)$ & 40,08 \\
\hline Angulo et al. (2015) & Calostrómetro (mg/ml) & 72.98 \\
\hline Bartier et al. (2015) & Calostrómetro & 82,3 \\
\hline Bartier et al. (2015) & $\mathrm{RID}\left(\mathrm{mg} \mathrm{ml}^{-1}\right)$ & 63,7 \\
\hline Bartier et al. (2015) & $\operatorname{Brix}(\%)$ & 24,3 \\
\hline Bielmann et al. (2010) & Brix $(\%)$ & 22 \\
\hline Bielmann et al. (2010) & $\operatorname{RID}\left(\mathrm{mg} \mathrm{ml}^{-1}\right)$ & 91,8 \\
\hline Morrill et al. (2012) & $\mathrm{RID}\left(\mathrm{mg} \mathrm{ml}^{-1}\right)$ & 71,5 \\
\hline Mann et al. (2016) & $\operatorname{RID}\left(\mathrm{mg} \mathrm{ml}^{-1}\right)$ & 96,1 \\
\hline Thu-Hang et al. (2017) & $\operatorname{Brix}(\%)$ & 35,6 \\
\hline Santos et al. (2017) & Calostrómetro & $51,7 \pm 8,11$ \\
\hline Elsohaby et al. (2016) & $\operatorname{IRS}\left(\mathrm{g} \mathrm{l}^{-1}\right)$ & 47,4 \\
\hline
\end{tabular}

RID: inmunodifusión radial. ELISA: inmunoabsorción ligada a enzimas. IR: espectroscopía de infrarrojo. IgG: inmunoglobulina G / RID: radial immunodiffusion. ELISA: enzyme-linked immunosorbent. IR: infrared spectroscopy. IgG: immunoglobulin G.

los valores de IgG fueron más bajos por ELISA en las muestras sometidas a tratamiento térmico (Gelsinger et al., 2015). Es preciso recalcar, respecto al tratamiento térmico del calostro, el uso de este método para garantizar la calidad higiénica sin afectar la concentración de IgG (Salazar-Acosta y Elizondo-Salazar, 2019).

Con relación a la espectroscopía de transmisión infrarroja (IRS), es una técnica rápida que requiere una mínima preparación de las muestras, y que permite medir concentraciones moleculares en fluidos biológicos, y puede usarse un solo espectro para el análisis cuantitativo de diversos componentes. Con el fin de evaluar la concentración de IgG en calostro de bovinos, se desarrolló un método analítico que resultó efectivo para dicha cuantificación y podría usarse para medir la calidad de calostro (Elsohaby et al., 2016).

Referente a ensayos enzimáticos, se probó una técnica molecular basada en la medición directa de $\operatorname{IgG}$ tanto en suero sanguíneo como en calostro, con una prueba denominada ensayo de inmunoglobulina $\mathrm{G}$ de trehalasa dividida (STIGA). Los autores hallaron una alta sensibilidad, especificidad y exactitud de la técnica comparada con otros métodos como RID, validando dicho método como otra alternativa para medir IgG (Drikic et al., 2018).

Las técnicas de laboratorio proveen valores más precisos de $\mathrm{IgG}$, debido a los esquemas productivos y a la necesidad de pruebas prácticas en campo, pero las técnicas de uso más común son el calostrómetro y la refractometría. En cuanto al calostrómetro, consiste en un dispositivo que mide la gravedad específica del calostro en $\mathrm{mg} \mathrm{ml}^{-1}$, y provee una estimación de la calidad relativa, pero no indica la concentración precisa de IgG (Elsohaby et al., 2016). El calostrómetro se ve afectado no solo por la IgG, sino por la temperatura del calostro y el contenido de solidos totales, además de ser un dispositivo frágil, que requiere ser limpiado cuidadosamente (Bielmann et al., 2010; Bartier et al., 2015).

La refractometría (óptica o digital) mide los grados Brix y/o la concentración de proteína total (TPR), que hace referencia principalmente a la cantidad de IgG (Elsohaby et al., 2017). Su uso generalizado se debe a que diversos estudios reportan una fuerte correlación, entre los grados Brix y la concentración de IgG (Bielmann et al., 2010; Bartier et al., 2015). 
A partir de las concentraciones de IgG en plasma y del consumo de las mismas por parte del ternero, se puede evaluar la eficiencia en la absorción de estas, y saber si el animal tuvo un adecuado nivel de ingesta de calostro. Esto se puede calcular con la ecuación 1 (Foster et al., 2016):

$$
\frac{\text { plasma } \operatorname{Ig} G\left(g l^{-1}\right) \times \text { volumen de plasma }(l)}{\operatorname{Ig} G \text { consumidas }(g)} \times 100
$$

Respecto a la medición del calostro y el adecuado nivel de IgG, Godden et al. (2019), propusieron la siguiente guía presentada en el Cuadro 3 para considerar la calidad del mismo con base en la concentración de IgG.

Cuadro 3. Categorías propuestas para considerar la calidad del calostro, con base en la concentración de inmunoglobulina G (IgG). Colombia, 2019.

Table 3. Proposed categories to evaluate colostrum quality, based on the concentration of immunoglobulin G (IgG). Colombia, 2019.

\begin{tabular}{cccc}
\hline & & \multicolumn{2}{c}{ Niveles equivalentes } \\
\cline { 3 - 4 } Categorías propuestas & Niveles propuestos IgG $\left(\mathbf{g ~ l}^{-1}\right)$ & CalostroSTP $\left(\mathbf{g ~ d l}^{-1}\right)$ & Suero sanguíneo Brix $(\%)$ \\
\hline Excelente & $>25,0$ & $>6,2$ & $>9,4$ \\
Bueno & $18,0-24,9$ & $5,8-6,1$ & $8,9-9,3$ \\
Regular & $10,0-19,9$ & $5,1-5,7$ & $8,1-8,8$ \\
Malo & $<10$ & $<5,1$ & $<8,1$ \\
\hline
\end{tabular}

Adaptado de: Godden et al. (2019). STP: concentración de proteínas totales / Adapted from: Godden et al. (2019). STP: serum total protein.

Si se garantiza un buen encalostrado de las crías, se garantiza una buena inmunidad y una buena sanidad de las mismas. Garantizar un buen suministro de calostro en las primeras horas, contribuirá a disminuir el riesgo en la presentación de estas afecciones (Morrill et al., 2012). Las infecciones entéricas y respiratorias son las principales patologías en neonatos, y dependiendo del sistema de alojamiento se puede aumentar o disminuir la prevalencia de algunas patologías.

En corrales compartidos, se incrementa el riesgo de afecciones del tracto respiratorio, se disminuye la prevalencia de diarreas no infecciosas y se favorece el cambio de lactante a desteto, conllevando a mejores pesos post-destete (Svensson et al., 2003). En los corrales, los terneros tienen la capacidad de modificar su comportamiento en respuesta a su entorno, dedican más tiempo al consumo de alimento y tienen una mayor ganancia de peso que los animales alojados individualmente (Svensson y Liberg, 2006). El mayor consumo se ha atribuido a que los terneros pueden recurrir a imitar el comportamiento de otros animales, a diferencia de los que se alojan individualmente, que deben desarrollar un proceso propio de aprendizaje (De-Paula-Vieira et al., 2010; Costa et al., 2015).

En cuanto al alojamiento en corrales individuales, se ha sugerido que, la crianza de terneros bajo este método podría disminuir el riesgo de contagio de patologías en neonatos. Sin embargo, para que dicho método sea exitoso, hay que tener en cuenta que es necesario no solo restringir la movilidad, sino minimizar la circulación de aire entre compartimentos, de lo contrario, dicho manejo tendría una repercusión negativa en la salud, e incrementaría la prevalencia de enfermedades respiratorias. 


\section{Alimentación líquida con leche o lactoreemplazador}

Cuando nacen los terneros se enfrentan a un cambio en la fuente de alimentación, pasando de una nutrición placentaria a una alimentación principalmente láctea. Este cambio requiere adaptaciones fisiológicas y digestivas acordes a las nuevas condiciones dietarías. El funcionamiento de la maquinaria enzimática a nivel gastrointestinal para la digestión del alimento lácteo es una de esas adaptaciones (Guilloteau et al., 2009). Enzimas como la quimosina, la pepsina y la elastasa II pancreática en abomaso, así como la lactasa en intestino, responsables de la coagulación del alimento lácteo, la digestión y la absorción de los nutrientes, aumentan su concentración durante las primeras semanas de vida, disminuyendo gradualmente a medida que se alcanza el desarrollo funcional del rumen (Guilloteau et al., 2005).

En la alimentación líquida de los terneros durante la crianza, se utiliza leche entera o lactoreemplazadores. Estos últimos son de preferencia por los menores costos, y porque se puede controlar mejor la composición del suplemento lácteo que se ofrece a los animales (Sharp, 2017). No obstante, la pertinencia de usar un lactoreemplazador o leche entera, depende del acceso a un buen producto en términos de economía, calidad nutricional y sanitaria (Krishnamoorthy y Moran, 2011).

Respecto al lactoreemplazador, su calidad varía entorno a la fuente de proteína y energía que contenga. En cuanto a la proteína, la fuente puede ser de origen animal o vegetal, siendo la primera de mejor calidad debido al perfil de aminoácidos, a su mejor digestibilidad y a la ausencia de fibra (Krishnamoorthy y Moran, 2011). En los lactoreemplazadores cuya proteína es de origen vegetal, esta puede provenir de la torta de soja, el gluten de maíz u otras fuentes, y su calidad puede diferir mucho más, porque a pesar de la alta calidad que puedan tener las materias primas, sus aportes de fibra pueden llegar a ser desfavorables en el alimento (American Feed Industry Association, 1995; Terui et al., 1996).

La proteína de origen animal de los lactoreemplazadores, proviene principalmente de suero seco, concentrado de proteína de suero, caseína, caseinato de sodio o leche descremada, siendo las proteínas de la leche (más específicamente las proteínas del suero de leche) la fuente principal. Las proteínas de la leche se consideran una buena alternativa, debido a la falta de factores antinutricionales y a una alta digestibilidad, que permite una tasa de crecimiento similar a la de los terneros que consumen la leche de su madre; además, representa un menor costo que el uso de leche entera (American Feed Industry Association, 1995). Sin embargo, el costo del lactoreemplazador sigue siendo motivo de investigación, en búsqueda de alternativas. El uso de una mezcla de proteína plasmática bovina y proteína de trigo modificada, se evaluó como reemplazo parcial de la proteína de suero del lactoreemplazador, y los resultados indicaron que el desempeño de los terneros fue similar a los que consumieron leche entera, con un menor costo (Sharp, 2017).

Con relación a la energía del lactoreemplazador, esta es una fuente de variación significativa en la calidad, ya que podría tener niveles superiores de lactosa (42-45\%) y a su vez, menor porcentaje de grasa (16-20\% en base seca) que la leche entera (30\% en base seca) (Park, 2009; Amado et al., 2019). Además, la leche entera contiene cerca de 5,21 Mcal EM kg-1 MS, y los lactoreemplazadores comerciales podrían tener en su mayoría un rango entre 4,16 y 4,79 Mcal EM kg-1 MS, lo que indica que los últimos podrían proveer menor cantidad de energía metabolizable al suministrarse en igual cantidad (Cuadro 4).

Diferentes estrategias han sido utilizado para compensar las diferencias en el suministro de energía entre lactoreemplazador y leche entera, como tasas de suministro más altas del lactoreemplazador, o la modificación de su composición para aumentar ingesta de nutrientes (Silva-Del-Río et al., 2017).

Con respecto a la composición del lactoreemplazador, no se encontraron diferencias significativas al comparar el crecimiento y desarrollo de terneros, utilizando un lactoreemplazador alto en grasa con uno alto en lactosa (Amado et al., 2019). Tampoco se encontraron diferencias significativas en el crecimiento y desarrollo de machos Holstein, cuando se evaluó un lactoreemplazador con $75 \%$ de leche descremada y $25 \%$ de insumos vegetales 
Cuadro 4. Valores promedio reportados para diferentes lactoreemplazadores y leche como porcentaje de la materia seca. Colombia, 2019.

Table 4. Average values reported for different milk replacers and milk as a percentage of the dry matter. Colombia, 2019.

\begin{tabular}{|c|c|c|c|c|c|c|c|c|c|}
\hline Dieta & Autor & $\operatorname{MS}(\%)$ & $\begin{array}{l}\text { Grasa (g/ } \\
\text { 100gMS) }\end{array}$ & $\begin{array}{c}\text { PC (g/ } \\
\text { 100gMS) }\end{array}$ & $\begin{array}{c}\text { Lactosa (g/ } \\
\text { 100gMS) }\end{array}$ & $\mathrm{Ca}$ & $\mathbf{P}$ & Cenizas & $\begin{array}{c}\text { EM (Mcal } \\
\left.\mathbf{k g}^{-1}\right)\end{array}$ \\
\hline \multirow{8}{*}{$\begin{array}{l}\text { Lactoreempla- } \\
\text { zador }\end{array}$} & Silper et al.(2014) & 94,6 & 17 & 20,7 & NR & 2,88 & 0,76 & 9,7 & 4,16 \\
\hline & Amado et al. (2019) & 96,8 & 17,3 & 23,2 & 44,3 & 8,8 & 6,9 & NR & 4,4 \\
\hline & Dennis et al. (2019) & 96,3 & 18,3 & 24,8 & NR & NR & NR & 5,58 & NR \\
\hline & Hill et al. (2009) & 96,3 & 31 & 27,3 & NR & 0,75 & 0,67 & 7,4 & 5,44 \\
\hline & Quigley et al.(2019) & 96,9 & 17,6 & 25,8 & 51 & 0,73 & 0,62 & 5,6 & NR \\
\hline & Raeth et al. (2016) & 97,42 & 20,12 & 20,98 & NR & 0,94 & 0,98 & 9,34 & NR \\
\hline & $\begin{array}{c}\text { Chapman et al. } \\
\text { (2016) }\end{array}$ & 96,2 & 21,2 & 21,1 & NR & NR & NR & 6,7 & 4,79 \\
\hline & $\begin{array}{l}\text { Berends et al. } \\
\quad(2016)\end{array}$ & NR & 21,3 & 20,8 & NR & 0,86 & 0,68 & 7,1 & NR \\
\hline \multirow{4}{*}{ Leche } & $\begin{array}{l}\text { Pacheco et al. } \\
\qquad(2016)\end{array}$ & 12,4 & 25,7 & 23,4 & NR & NR & NR & NR & NR \\
\hline & Silva et al. (2017) & 11,4 & 28,5 & 25,6 & NR & NR & NR & NR & NR \\
\hline & Hill et al. (2009) & 13,6 & 27,6 & 25,3 & NR & NR & NR & NR & 5,21 \\
\hline & Gulati et al. (2018) & NR & 48,5 & 39,7 & 47,2 & NR & NR & NR & NR \\
\hline
\end{tabular}

NR: No reporta. MS: materia seca. PC: proteína cruda. EM: energía metabolizable / NR: does not report. MS: dry matter. PC: crude protein. EM: metabolizable energy.

no convencionales (lupino, vicia, linaza y trigo), comparado con un tratamiento de leche entera y con otro lactoreemplazador con $80 \%$ de leche descremada y $20 \%$ de manteca de cerdo (LR-LDMC), pero sí se encontró una reducción significativa en los costos con el tratamiento que incluía insumos vegetales alternativos (Flores, 2019).

La cantidad de leche o lactoreemplazador y el tiempo de ofrecimiento a utilizar durante la crianza de terneros es un factor variable. Los resultados en el desempeño de los animales son diferentes porque hay diversos factores que inciden, entre ellos la cantidad y calidad del alimento sólido ofrecido y el momento de empezar a ofrecerlo. Sin embargo, los resultados investigativos parecieran indicar que un periodo de lactancia de ocho semanas podría ser el apropiado; no obstante, sería necesario evaluar bajo condiciones específicas, ya que hay estudios que indican que el destete en machos y hembras en lecherías, podría hacerse a las seis semanas o menos (Kehoe et al., 2007).

En relación con la evaluación del efecto de la duración de la fase de alimentación láctea, evaluaciones realizadas sobre el crecimiento de crías Holstein desde el nacimiento hasta un mes después del destete, en animales recibiendo calostro, leche, concentrado iniciador y heno de alfalfa durante 90 o 60 días, reportaron una mayor ganancia de peso diaria en los animales alimentados a los 60 días $\left(0,65 \mathrm{vs} 0,70 \mathrm{~kg}_{\text {animal }}{ }^{-1}\right.$ día $^{-1}, 90$ y 60 días, respectivamente) (Gutiérrez, 2000).

En la evaluación del crecimiento, antes y después del destete, de terneros lecheros Sahiwal destetados a las ocho o doce semanas, en las que se ofreció leche al $10 \%$ o $15 \%$ del peso corporal (BW), se encontró que la alimentación láctea durante ocho semanas y con alto contenido de leche (15\%), ahorró leche y mano de obra y no hubo diferencia en los pesos (Cheema et al., 2016).

En un ensayo, donde se caracterizó el efecto de la edad del destete (seis vs ocho semanas) en la ingesta de MS (leche, heno y alimento concentrado), en el crecimiento animal y el desarrollo del tracto digestivo, en machos 
Holstein, se encontró que los animales destetados a las ocho semanas tuvieron un mayor consumo de alimento sólido (mayor heno, menor alimento concentrado) y 9,8\% más de peso que el grupo destetado a las seis semanas $(91 \mathrm{~kg})$. Los terneros destetados a las ocho semanas, tuvieron una mejor transición de la leche a la alimentación sólida, en comparación con el grupo destetado a las seis semanas (Eckert et al., 2015). Los destetes de hembras y machos provenientes de lecherías, realizados a diferentes edades (3, 4, 5, 6, 8 semanas), con el suministro del lactoreemplazador con un 12,5\% de materia seca, $22 \%$ de proteína y 15,6 \% de grasa, a razón del $10 \%$ del peso vivo, no presentaron diferencias en el peso ni ganancia de peso, ni en el desarrollo ruminal, pero los terneros destetados a las tres semanas no tuvieron un buen consumo de alimento concentrado antes del destete, siendo el mejor consumo de concentrado pre y post destete, cuando se realizó el destete a la quinta y sexta semana. Esto podría indicar la posibilidad de hacer un destete efectivo antes de la semana ocho (Kehoe et al., 2007).

En cuanto a la cantidad de suplemento lácteo a suministrar, el protocolo que predomina es el de suministrar leche o lactoreemplazador de forma restringida durante el periodo de lactancia, con el fin de reducir costos en la crianza, estimular el consumo de alimento concentrado y promover el desarrollo temprano del rumen. Algunos protocolos incluyen aproximadamente cinco semanas de una cantidad determinada de alimento lácteo acompañado con suministro a voluntad de alimento sólido, y luego tres semanas de transición, donde la cantidad de alimento lácteo disminuye gradualmente (Schäff et al. (2016), mientras que otros consideran siete semanas (49 días) de suplementación láctea fija y dos semanas (14 d) de transición con reducción gradual (Amado et al., 2019).

Dentro de los protocolos restringidos para el suministro de leche en la crianza de machos Holstein, se reporta el abastecimiento de 5,5 1 día $^{-1}$ de leche hasta el día 56, y 31 día $^{-1}$ hasta el día 59, para un total de ocho semanas, y protocolos graduales con 71 día $^{-1}$ de leche hasta el día 35, 41 día ${ }^{-1}$ desde el día 35 hasta el día 49, y 21 día ${ }^{-1}$ desde el día 50 hasta el día 59 (Daneshvar et al., 2017).

Los protocolos restringidos han sido cuestionados porque se cree que, al suministrar alimento lácteo a voluntad, se logra una mejor condición sanitaria y desempeño animal (Ollivett et al., 2012; Maccari et al., 2015). A pesar de ello, se ha reportado una mejor respuesta suministrando leche de forma restringida, a razón del $15 \%$ del peso vivo de los terneros (Cheema et al., 2016), tampoco se han encontraron diferencias en el estado sanitario de terneros (machos y hembras Holstein y Holstein x Charoláis) restringidos vs ad libitum, durante ocho semanas, pero se halló una mayor eficiencia alimenticia en los animales restringidos, consumiendo 61 de lactoreemplazador animal $^{-1}$ día $^{-1}$ (Schäff et al., 2016).

En cuanto a la cantidad total de suministro de lactoreemplazador, se encontró que no se debe exceder el suministro por encima de $0,7 \mathrm{~kg}$ de materias seca día ${ }^{-1}$ en la etapa pre-destete, puesto que valores por encima, podrían ocasionar un desarrollo ruminal más bajo, debido a un bajo aporte de fibra en la dieta y esto podría comprometer la ganancia de peso post-destete, donde el animal necesita un buen desarrollo ruminal ya que su dieta tiene un gran contenido de fibra (Chapman et al., 2016).

Estos resultados contrastantes indican la necesidad de evaluar bajo condiciones tropicales, el uso de lactoreemplazadores o leche de forma restringida o ad libitum, sobre todo porque la calidad del lactoreemplazador, del alimento concentrado y del forraje, pueden ofrecer condiciones específicas que afecten los resultados. Se encontraron reportes donde hembras y machos Holstein que consumieron mayores cantidades de leche (hasta 8 1 día $^{-1}$ ) tuvieron significativamente menores consumos de alimento sólido, así como un menor desarrollo ruminal y crecimiento papilar, sin diferencias en las ganancias de peso (Elizondo-Salazar y Sánchez-Álvarez, 2012). Si se considera que los machos y hembras de descarte que se destinarán para producción de carne tendrán una dieta mayoritaria o netamente forrajera después del destete, el desarrollo y funcionamiento del rumen pre-destete, debe ser fundamental y de ahí, la importancia de dar cantidades controladas de alimento lácteo y estimular el consumo de alimento sólido. 


\section{Alimentación sólida}

En el neonato el rumen no se encuentra desarrollado ni física ni metabólicamente, además, no presenta el grado de queratinización característico del órgano maduro. Una vez que se inicia el consumo de alimento sólido y se establece la fermentación ruminal y consiguiente producción de ácidos grasos de cadena corta en el lumen ruminal, se generan una serie de cambios físicos y metabólicos (Gilliland et al., 2010).

El establecimiento de la microbiota incluye bacterias, hongos y protozoos, siendo las primeras, las responsables del proceso de fermentación del alimento sólido y la producción de los ácidos grasos volátiles (AGV’S), acetato, propionato y butirato. Estos AGV son la principal fuente energética del rumiante funcional (Jami et al., 2013), siendo los ácidos butírico y propiónico, los que se relacionan principalmente con el desarrollo en la longitud y el diámetro de las papilas ruminales, debido a que aceleran la tasa de mitosis, y disminuyen la apoptosis de las células del epitelio ruminal (Beiranvand et al., 2014; Govil et al., 2017), y en especial el butírico, porque es la fuente de energía principal de las células del epitelio y papilas ruminales (Suárez-Mena et al., 2017).

El cambio de una dieta líquida a inclusión de alimento sólido, altera la expresión de más de 900 transcripciones de genes relacionados con el metabolismo lipídico, la morfología, el crecimiento, la proliferación y la muerte celular, el transporte molecular y el ciclo celular (Connor et al., 2013).

La función de los alimentos sólidos es promover la transición de pre-rumiante a rumiante, estimulando cambios anatómicos, fisiológicos e histológicos importantes, requeridos para el adecuado funcionamiento del rumen (Guilloteau et al., 2009). Cambios de una digestión enzimática a fermentativa, están relacionados con la edad en que se inicia el suministro del alimento sólido, así como, con la calidad, la cantidad y la forma del mismo, puesto que, es la parte de la dieta que tiene una influencia directa sobre el desarrollo ruminal (Terré et al., 2013). Así, de acuerdo con el manejo nutricional que se realice del alimento sólido, se puede acelerar o retrasar el desarrollo ruminal completo, lo cual generalmente se espera que se alcance finalizando las doce semanas de vida (Jones y Heinrichs, 2016).

El suministro de alimento concentrado se recomienda desde la primera semana de vida de los terneros. La cantidad se incrementa de forma gradual, con el fin de que se logre un consumo de al menos $1 \mathrm{~kg}_{\text {animal }}{ }^{-1}$ día $^{-1}$ al destete a las ocho semanas, periodo en el que se espera dupliquen el peso al nacimiento, lo cual se considera una medida de eficiencia y adecuado manejo en la crianza (Elizondo-Salazar y Monge-Rojas, 2019). El contenido de proteína puede variar entre el 18 y el $24 \%$, sin embargo, Daneshvar et al. (2017) no encontraron diferencias en el crecimiento, desarrollo y salud de terneros suplementados con alimento concentrado con $20 \%$ o $24 \%$ de proteína durante la crianza.

La presentación física del alimento también podría tener influencia sobre el desarrollo del rumen, debido a que la alteración de la forma física o química del alimento, incrementa el área de absorción o aprovechamiento de los nutrientes. Animales que consumieron alimento extruido presentaron un menor desarrollo de las papilas y menor grosor de la pared ruminal, comparado con animales que consumieron alimento en harina, concluyendo que el molido o quebrado de los granos tiene mayor efecto sobre el desarrollo papilar, que los procesos térmicos que producen más propionato en la fermentación ruminal (Castro-Flores y Elizondo-Salazar, 2012).

Otro factor que se relaciona con el desarrollo ruminal, es la ingesta de material fibroso. Durante las últimas dos décadas, la alimentación de forraje no se ha recomendado para terneros de lecherías durante la cría (antes del destete). Sin embargo, cuando se permite el acceso al pasto desde las primeras semanas de vida, los terneros comienzan a comer pequeñas cantidades a temprana edad (Tedeschi and Fox, 2009), y esto permite que incrementen el consumo en la medida que disminuye la ingesta de leche hasta el destete.

Novillas lecheras criadas con una dieta de solo concentrado antes del destete, comieron menos forraje después del destete en comparación con terneros criados con forraje más concentrado antes del destete, probablemente debido a diferencias en las capacidades para ingerir y digerir el forraje (Khan et al., 2012). No obstante, si bien su ingesta promueve el desarrollo físico del rumen, un alto consumo de material fibroso, puede limitar la ingesta de alimentos altamente fermentables y verse reflejado en un retraso en el desarrollo del epitelio ruminal (Beiranvand 
et al., 2014). Esto sugiere que debe haber un equilibrio en la dieta, alimentos fibrosos y altamente fermentables y los alimentos fibrosos deben ser de muy buena calidad.

La suplementación con componentes altamente fibrosos y de baja calidad, interfieren con el normal desarrollo fisiológico del rumen, y hay una relación positiva entre la suplementación con fibras de buena calidad y el desarrollo del epitelio ruminal (Castells et al., 2012). La suplementación con fibra de calidad en terneros durante la fase de transición, se asocia con una mejor fermentación post-destete, comparado con animales sin suplementación fibrosa (Terré et al., 2013), hallazgos que coinciden con lo reportado por Nemati et al. (2016) y EbnAli et al. (2016), quienes asociaron una mejor funcionalidad del rumen con el consumo de fibra de calidad, debido principalmente al efectos físico de la misma sobre el desarrollo muscular del rumen.

Adicional a la calidad de la fibra, existen alternativas no convencionales para promover el desarrollo ruminal, relacionadas con la suplementación forrajera, como la suplementación con extractos de plantas, a modo de opción al uso de antibióticos como promotores de crecimiento (Macheboeuf et al., 2008). Sin embargo, en mayor medida, el uso de alternativas forrajeras y de alimentos sólidos altamente fermentables a temprana edad que promuevan el desarrollo ruminal, reduzcan el suministro de alimento lácteo (leche entera o lactoreemplazador), y con ello los trastornos digestivos durante esta etapa, se convierten en una alternativa relevante, puesto que podría representar una diminución en los costos de alimentación y mano de obra en el pre-destete, y un incremento en el margen de rentabilidad (Aquino y Cruz, 2019), sin dejar de lado el aporte de los requerimientos nutricionales, evitando así, el retraso en el crecimiento, desarrollo y potencial productivo de los animales (Byrne et al., 2017), influenciando positivamente la eficiencia alimenticia (Flores, 2019).

\section{Conclusiones}

La crianza de machos y hembras de descarte provenientes de las lecherías especializadas, requiere de un manejo minucioso de la alimentación como etapa crítica en la producción de carne.

El suministro de calostro de calidad en el momento apropiado y en la cantidad requerida, es fundamental para garantizar el éxito de toda la etapa productiva debido a que garantiza no solo la sanidad del animal sino también la maduración y función neonatal del tracto gastrointestinal, influenciando directamente los parámetros de mortalidad durante las diferentes etapas del desarrollo.

La alimentación líquida puede estar compuesta por leche entera o lactoreemplazador, el suministro de uno u otro dependerá de los costos, la calidad y las condiciones sanitarias, entre otros, y se deberá procurar porque por acortar el tiempo de suministro en pro de mejorar el margen de utilidad, al suministrar cantidades apropiadas que no afecten el consumo de alimento concentrado ni el desarrollo ruminal, no sobrepasando los $0,7 \mathrm{~kg} \mathrm{MS} \mathrm{día}{ }^{-1} \mathrm{ni}$ las ocho semanas de suministro lácteo.

El suministro de alimento sólido, concentrado y forraje, y la calidad de estos, incluyendo un adecuado equilibrio en el contenido de fibra y carbohidratos solubles, es fundamental en el manejo de la alimentación, ya que de esto dependerá el tiempo que transcurra hasta alcanzar el desarrollo ruminal completo y la capacidad para sostener una dieta forrajera post-destete.

\section{Agradecimientos}

Los autores agradecen a la Universidad de Antioquia y a la Cooperativa Colanta por el apoyo financiero al proyecto "Caracterización y evaluación bio-económica de la cría de terneros provenientes de lechería especializada, con destino futuro a la producción de carne en trópico alto" y a la primera autora para el desarrollo de su maestría a través de una beca. 


\section{Literatura citada}

Amado, L., H. Berends, L.N. Leal, J. Wilms, H. Van-Laar, W.J.J. Gerrits, and J. Martín-Tereso. 2019. Effect of energy source in calf milk replacer on performance, digestibility, and gut permeability in rearing calves. J. Dairy Sci. 102:3994-4001. doi:10.3168/jds.2018-15847

American Feed Industry Association. 1995. A guide to colostrum and colostrum management for dairy calves. USDA, USA.

Angulo, J., L.M. Gómez, L. Mahecha, E. Mejía, J. Henao, and C. Mesa. 2015. Calf's sex, parity and the hour of harvest after calving affect colostrum quality of dairy cows grazing under high tropical conditions. Trop. Anim. Health Prod. 47:699705. doi:10.1007/s11250-015-0781-z

Aquino, J., y A. Cruz. 2019. Efecto de la suplementación con dos pre-iniciadores sobre el desempeño productivo de terneras Holstein. Tesis Lic., Escuela Agrícola Panamericana, Zamorano, HON.

Bartier, A.L., M.C. Windeyer, and L. Doepel. 2015. Evaluation of on-farm tools for colostrum quality measurement. J. Dairy Sci. 98:1878-1884. doi:10.3168/jds.2014-8415

Beiranvand, H., G.R. Ghorbani, M. Khorvash, A. Nabipour, and A. Homayouni. 2014. Interactions of alfalfa hay and sodium propionate on dairy calf performance and rumen development. J. Dairy Sci. 97:2270-2280. doi:10.3168/jds.2012-6332

Bielmann, V., J. Gillan, N.R. Perkins, A.L. Skidmore, S. Godden, and K.E. Leslie. 2010. An evaluation of Brix refractometry instruments for measurement of colostrum quality in dairy cattle. J. Dairy Sci. 93:3713-3721 . doi:10.3168/jds.2009-2943

Byrne, C.J., S. Fair, A.M. English, D. Johnston, P. Lonergan, and D.A. Kenny. 2017. Effect of milk replacer and concentrate intake on growth rate, feeding behaviour and systemic metabolite concentrations of pre-weaned bull calves of two dairy breeds. Animal 11:1531-1538. doi: 10.1017/S1751731117000350

Castells, L., A. Bach, G. Araujo, C. Montoro, and M. Terré. 2012. Effect of different forage sources on performance and feeding behavior of Holstein calves. J. Dairy Sci. 95:286-293. doi:10.3168/jds.2011-4405

Castro-Flores, P., y J.A. Elizondo-Salazar. 2012. Crecimiento y desarrollo ruminal en terneros alimentados con iniciador sometido a diferentes procesos. Agron. Mesoam. 23:343-352. doi:10.15517/AM.V23I2.6534

Chapman, C.E., P.S. Erickson, J.D. Quigley, T.M. Hill, H.G. Bateman, F.X. Suarez-Mena, and R.L. Schlotterbeck. 2016. Effect of milk replacer program on calf performance and digestion of nutrients with age of the dairy calf. J. Dairy Sci. 99:27402747. doi:10.3168/jds.2015-10372

Cheema, A.T., S.A. Bhatti, G. Akbar, P.C. Wynn, G. Muhammad, H.M. Warriah, and D. McGill. 2016. Effect of weaning age and milk feeding level on pre- and post-weaning growth performance of Sahiwal calves. Anim. Prod. Sci. 58:314-321. doi:10.1071/AN15719

Conneely, M., D.P. Berry, R. Sayers, J.P. Murphy, I. Lorenz, M.L. Doherty, and E. Kennedy. 2013. Factors associated with the concentration of immunoglobulin G in the colostrum of dairy cows. Animal 7:1824-1832. doi:10.1017/S1751731113001444

Connor, E.E., R.L.B. Vi, C. Li, R.W. Li, and H. Chung. 2013. Gene expression in bovine rumen epithelium during weaning identifies molecular regulators of rumen development and growth. Funct. Integr. Genomics 13:133-142. doi:10.1007/ s10142-012-0308-x

Costa, J.H.C., R.K. Meagher, M.A.G. von-Keyserlingk, and D.M. Weary. 2015. Early pair housing increases solid feed intake and weight gains in dairy calves. J. Dairy Sci. 98:6381-6386. doi:10.3168/jds.2015-9395

Daneshvar, D., M. Khorvash, E. Ghasemi, and A.H. Mahdavi. 2017. Combination effects of milk feeding methods and starter crude protein concentration: Evaluation on performance and health of Holstein male calves. Anim. Feed Sci. Technol. 223:1-12. doi:10.1016/j.anifeedsci.2016.10.025 
De-Paula-Vieira, A., M.A.G. von-Keyserlingk, and D.M. Weary. 2010. Effects of pair versus single housing on performance and behavior of dairy calves before and after weaning from milk. J. Dairy Sci. 93:3079-3085. doi:10.3168/jds.2009-2516

Dichio, L., L. Amprimo, C. Azzaro, L. Almirón, G. Puccio, y J. Galli. 2015. Crianza artificial de las terneras en el Módulo de Producción Lechera de la Facultad de Ciencias Agrarias. Agromensajes 42:47-50.

dos-Santos, G., J.T. da-Silva, F.H. Santos, and C.M. Bittar. 2017. Nutritional and microbiological quality of bovine colostrum samples in Brazil. R. Bras. Zootec. 46:72-79. doi:10.1590/s 1806-92902017000100011

Drikic, M., C. Windeyer, S. Olsen, Y. Fu, L. Doepel, and J. De-Buck. 2018. Determining the IgG concentrations in bovine colostrum and calf sera with a novel enzymatic assay. J. Anim. Sci. Biotechnol. 9:69. doi:10.1186/s40104-018-0287-4

Dunn, A., A. Ashfield, B. Earley, M. Welsh, A. Gordon, and S.J. Morrison. 2017. Evaluation of factors associated with immunoglobulin G, fat, protein, and lactose concentrations in bovine colostrum and colostrum management practices in grassland-based dairy systems in Northern Ireland. J. Dairy Sci. 100:2068-2079. doi:10.3168/jds.2016-11724

EbnAli, A., M. Khorvash, G.R. Ghorbani, A.H. Mahdavi, M. Malekkhahi, M. Mirzaei, A. Pezeshki, and M.H. Ghaffari. 2016. Effects of forage offering method on performance, rumen fermentation, nutrient digestibility and nutritional behaviour in Holstein dairy calves. J. Anim. Physiol. Anim. Nutr. (Berl). 100:820-827. doi:10.1111/jpn.12442

Eckert, E., H.E. Brown, K.E. Leslie, T.J. DeVries, and M.A. Steele. 2015. Weaning age affects growth, feed intake, gastrointestinal development, and behavior in Holstein calves fed an elevated plane of nutrition during the preweaning stage. J. Dairy Sci. 98:6315-6326. doi: 10.3168/jds.2014-9062

Elizondo-Salazar, J.A., and C.R. Monge-Rojas. 2019. Consumo de alimento balanceado en reemplazos de lechería desde el nacimiento hasta las 8 semanas de edad. Nutr. Anim. Trop. 13(2):58-75. doi:10.15517/nat.v13i2.39698

Elizondo-Salazar, J.A., y M. Sánchez-Álvarez. 2012. Efecto del consumo de dieta líquida y alimento balanceado sobre el crecimiento y desarrollo ruminal en terneras de lechería. Agron. Costarricense 36(2):81-90.

Elizondo-Salazar, J.A., y H.A. Solís-Chaves. 2018. Costo de criar una ternera lechera de reemplazo desde el nacimiento al parto. Agron. Mesoam. 29:547-555. doi:10.15517/ma.v29i3.32545.

Elsohaby, I., M. Claire Windeyer, D.M. Haines, E.R. Homerosky, J.M. Pearson, J.T. McClure, and G.P. Keefe. 2018. Application of transmission infrared spectroscopy and partial least squares regression to predict immunoglobulin g concentration in dairy and beef cow colostrum. J. Anim. Sci. 96:771-782. doi:10.1093/jas/sky003

Elsohaby, I., J.T. McClure, M. Cameron, L.C. Heider, and G.P. Keefe. 2017. Rapid assessment of bovine colostrum quality: How reliable are transmission infrared spectroscopy and digital and optical refractometers? J. Dairy Sci. 100:1427-1435. doi:10.3168/jds.2016-11824

Elsohaby, I., J.T. McClure, S. Hou, C.B. Riley, R.A. Shaw, and G.P. Keefe. 2016. A novel method for the quantification of bovine colostral immunoglobulin G using infrared spectroscopy. Int. Dairy J. 52:35-41. doi:10.1016/j.idairyj.2015.08.004

Espada, M., J.J. Ramos, L.M. Ferrer, A. Loste, A. Ortín, y A. Fernández. 2011. El calostro. clave de supervivencia. Servet, ESP.

Flores, R. 2019. Evaluación de un lacto reemplazante con insumos vegetales no tradicionales en becerros Holstein cruzados. Tesis MSc., Universidad Nacional de Cajamarca, Cajamarca, PER.

Foster, D.M., K.P. Poulsen, H.J. Sylvester, M.E. Jacob, K.E. Casulli, and B.E. Farkas. 2016. Effect of high-pressure processing of bovine colostrum on immunoglobulin $\mathrm{G}$ concentration, pathogens, viscosity, and transfer of passive immunity to calves. J. Dairy Sci. 99:8575-8588. doi:10.3168/jds.2016-11204

Gavin, K., H. Neibergs, A. Hoffman, J.N. Kiser, M.A. Cornmesser, S.A. Haredasht, B. Martínez-López, J.R. Wenz, and D.A. Moore. 2018. Low colostrum yield in Jersey cattle and potential risk factors. J. Dairy Sci. 101:6388-6398. doi:10.3168/ jds.2017-14308 ISSN 2215-3608 doi:10.15517/am.v31i3.40217 
Gelsinger, S.L., A.M. Smith, C.M. Jones, and A.J. Heinrichs. 2015. Technical note: Comparison of radial immunodiffusion and ELISA for quantification of bovine immunoglobulin G in colostrum and plasma. J. Dairy Sci. 98:4084-4089. doi:10.3168/ jds.2014-8491

Gilliland, R.L., L.J. Bush, and J.D. Friend. 2010. Relation of ration composition to rumen development in Early-Weaned dairy calves with observations on ruminal parakeratosis. J. Dairy Sci. 45:1211-1217. doi:10.3168/jds.s0022-0302(62)89598-8

Godden, S.M., J.E. Lombard, and A.R. Woolums. 2019. Colostrum management for dairy calves. Vet. Clin. North Am. Food Anim. Pract. 35:535-556. doi:10.1016/j.cvfa.2019.07.005

Gómez, L.M., S.L. Posada, M. Olivera, R. Rosero, y P. Aguirre. 2017. Análisis de rentabilidad de la producción de leche de acuerdo con la variación de la fuente de carbohidrato utilizada en el suplemento de vacas holstein. Rev. Med. Vet. 34(Supl.):9-22. doi:10.19052/mv.4251

Gonsolin, R. 2013. ¿Cómo hacer negocio con el engorde de machos Holando? Dietas simples y eficientes. Engormix. https:// www.engormix.com/ganaderia-carne/articulos/como-hacer-negocio-con-t30397.htm (consultado 12 nov. 2019).

Govil, K., D.S. Yadav, A.K. Patil, S. Nayak, R.P.S. Baghel, P.K. Yadav, C.D. Malapure, and D. Thakur. 2017. Feeding management for early rumen development in calves. J. Entomol. Zool. Stud. 5:1132-1139.

Guilloteau, P., R. Zabielski, and P.C. Garnsworthy. 2005. Digestive secretions in preruminant and ruminant calves and some aspects of their regulation. In: P.C. Garnsworthy, editor, Calf and heifer rearing. University Press, Nottingham, GBR. p. 159-189.

Guilloteau, P., R. Zambielski, and J. Blum. 2009. Gastrointestinal tract and digestion in the young ruminant: Ontogenesis, adaptations, consequences and manipulations. J. Physiol. Pharmacol. 60:37-46.

Gulati, A., N. Galvin, E. Lewis, D. Hennessy, M. O’Donovan, J.J. McManus, M.A. Fenelon, and T.P. Guinee. 2018. Outdoor grazing of dairy cows on pasture versus indoor feeding on total mixed ration: Effects on gross composition and mineral content of milk during lactation. J. Dairy Sci. 101:2710-2723. doi:10.3168/jds.2017-13338

Gutiérrez, W. 2000. Efecto de un plan alimenticio sobre el crecimiento de hembras Holstein Friesian, desde el nacimiento hasta un mes post destete. Tesis de Médico Veterinario. Univ. Nacional de Cajamarca, Cajamarca, PER.

Inagaki, M., H. Muranishi, K. Yamada, K. Kakehi, K. Uchida, T. Suzuki, Y. Yabe, T. Nakagomi, O. Nakagomi, and Y. Kanamaru. 2014. Bovine K-casein inhibits human rotavirus (HRV) infection via direct binding of glycans to HRV. J. Dairy Sci. 97:2653-2661. doi:10.3168/jds.2013-7792

Jami, E., A. Israel, A. Kotser, and I. Mizrahi. 2013. Exploring the bovine rumen bacterial community from birth to adulthood. ISME J. 7:1069-1079. doi:10.1038/ismej.2013.2

Jones, C., and J. Heinrichs. 2016. Early weaning strategies. PennState Extention, USA. https://extension.psu .edu/early-weaningstrategies (accessed May 3, 2020).

Kehoe, S.I., C.D. Dechow, and A.J. Heinrichs. 2007. Effects of weaning age and milk feeding frequency on dairy calf growth, health and rumen parameters. Livest. Sci. 110:267-272. doi:10.1016/j.livsci.2006.11.007

Kehoe, S.I., A.J. Heinrichs, M.L. Moody, C.M, Jones, and M.R. Long. 2011. Comparison of IgG concentration in primiparous and multiparous bovine colostrum. Prof. Anim. Sci. 27:176-180. doi:10.15232/S1080-7446(15)30471-X

Khan, M.A., D.M. Weary, D.M. Veira, and M.A.G. von-Keyserlingk. 2012. Postweaning performance of heifers provided hay during the milk feeding period. J. Dairy Sci. 95:3970-3976. doi:10.3168/jds.2011-5027

Krishnamoorthy, U., and J. Moran. 2011. Rearing young ruminants on milk replacers and starter feeds. FAO, Rome, ITA.

Maccari, P., S. Wiedemann, H.J. Kunz, M. Piechotta, P. Sanftleben, and M. Kaske. 2015. Effects of two different rearing protocols for Holstein bull calves in the first 3 weeks of life on health status, metabolism and subsequent performance. J. Anim. Physiol. Anim. Nutr. 99:737-746. doi:10.1111/jpn.12241 
Macheboeuf, D., D.P. Morgavi, Y. Papon, J.L. Mousset, and M. Arturo-Schaan. 2008. Dose-response effects of essential oils on in vitro fermentation activity of the rumen microbial population. Anim. Feed Sci. Technol. 145:335-350. doi:10.1016/j. anifeedsci.2007.05.044

Mann, S., F.A. Leal Yepes, T.R. Overton, A.L. Lock, S.V. Lamb, J.J. Wakshlag, and D.V. Nydam. 2016. Effect of dry period dietary energy level in dairy cattle on volume, concentrations of immunoglobulin G, insulin, and fatty acid composition of colostrum. J. Dairy Sci. 99:1515-1526. doi:10.3168/jds.2015-9926

McCracken, M.M., K.M. Morrill, A.L. Fordyce, and H.D. Tyler. 2017. Technical note: Evaluation of digital refractometers to estimate serum immunoglobulin G concentration and passive transfer in Jersey calves. J. Dairy Sci. 100:8438-8442. doi:10.3168/jds.2017-12847

McGuirk, S.M., and M. Collins. 2004. Managing the production, storage, and delivery of colostrum. Vet. Clin. North Am. Food Anim. Pract. 20:593-603. doi:10.1016/j.cvfa.2004.06.005

Ministerio de Agricultura, y Fundación para la Innovación Agraria. 2010. Producción de carne de ternero bajo un sistema asociativo. Pecuario / Bovinos 109. Ministerio de Agricultura, y Fundación para la Innovación Agraria, CHL.

Morrill, K.M., E. Conrad, A. Lago, J. Campbell, J. Quigley, and H. Tyler. 2012. Nationwide evaluation of quality and composition of colostrum on dairy farms in the United States. J. Dairy Sci. 95:3997-4005. doi:10.3168/jds.2011-5174

Nemati, M., H. Amanlou, M. Khorvash, M. Mirzaei, B. Moshiri, and M.H. Ghaffari. 2016. Effect of different alfalfa hay levels on growth performance, rumen fermentation, and structural growth of Holstein dairy calves. J. Anim. Sci. 94:1141-1148. doi:10.2527/jas.2015-0111

Ollivett, T., D. Nydam, T. Linden, D. Bowman, and M. Van-Amburgh. 2012. Effect of nutritional plane on health and performance in dairy calves after experimental infection with Cryptosporidium parvum. J. Am. Vet. Med. Assoc. 241:1514-1520. doi:10.2460/javma.241.11.1514.

Park, Y.W. 2009. Overview of bioactive components in milk and dairy products. In: Y.W. Park, editor, Bioactive components in milk and dairy products. Wiley-Blackwell, USA. p. 1-12. doi:10.1002/9780813821504.ch1

Pechova, A., S. Slosarkova, S. Stanek, E. Nejedla, and P. Fleischer. 2019. Evaluation of colostrum quality in the Czech Republic using radial immunodiffusion and different types of refractometers. Veterinárdní Medicína 64(2):51-59. doi:10.17221/122/2018-VETMED

Quigley, J.D., L. Deikun, T.M. Hill, F.X. Suarez-Mena, T.S. Dennis, and W. Hu. 2019. Effects of colostrum and milk replacer feeding rates on intake, growth, and digestibility in calves. J. Dairy Sci. 102:11016-11025. doi:10.3168/jds.2019-16682

Raeth, M., D. Ziegler, B. Ziegler, D. Schimek, and D.L. Cook. 2016. Pre- and postweaning performance and health of dairy calves fed milk replacers with differing protein sources. Prof. Anim. Sci. 32:833-841. doi:10.15232/pas.2016-01536

Salazar-Acosta, E., y J.A. Elizondo-Salazar. 2019. El tratamiento térmico del calostro aumenta la absorción de inmunoglobulinas G en terneras Holstein. Agron. Mesoam. 30:229-238. doi:10.15517/am.v30i1.32356

Schäff, C.T., J. Gruse, J. Maciej, M. Mielenz, E. Wirthgen, A. Hoeflich, M. Schmicke, R. Pfuhl, P. Jawor, T. Stefaniak, and H.M. Hammon. 2016. Effects of feeding milk replacer and libitum or in restricted amounts for the first five weeks of life on the growth, metabolic adaptation, and immune status of newborn calves. PLoS One 11:e0168974. doi:10.1371/journal. pone. 0168974

Sharp, K.D. 2017. The effect of feeding frequency and alternative proteins in milk. MSc. Thesis, University of Illinois, IL, USA.

Shivley, C.B., J.E. Lombard, N.J. Urie, D.M. Haines, R. Sargent, C.A. Kopral, T.J. Earleywine, J.D. Olson, and F.B. Garry. 2018. Preweaned heifer management on US dairy operations : Part II. Factors associated with colostrum quality and passive transfer status of dairy heifer calves. J. Dairy Sci. 101:9185-9198. doi:10.3168/jds.2017-14008 ISSN 2215-3608 doi:10.15517/am.v31i3.40217 
Silper, B.F., A.M.Q. Lana, A.U. Carvalho, C.S. Ferreira, A.P.S. Franzoni, J.A.M. Lima, H.M. Saturnino, R.B. Reis, and S.G.Coelho. 2014. Effects of milk replacer feeding strategies on performance, ruminal development, and metabolism of dairy calves. J. Dairy Sci. 97:1016-1025. doi:10.3168/jds.2013-7201

Silva, A.L., M.I. Marcondes, E. Detmann, M.M. Campos, F.S. Machado, S.C. Valadares-Filho, M.M.D. Castro, and J. Dijkstra. 2017. Determination of energy and protein requirements for crossbred Holstein $\times$ Gyr preweaned dairy calves. J. Dairy Sci. 100:1170-1178. doi:10.3168/jds.2016-11197

Silva-Del-Río, N., D. Rolle, A. García-Muñoz, S. Rodríguez-Jiménez, A. Valldecabres, A. Lago, and P. Pandey. 2017. Colostrum immunoglobulin G concentration of multiparous Jersey cows at first and second milking is associated with parity, colostrum yield, and time of first milking, and can be estimated with Brix refractometry. J. Dairy Sci. 100:5774-5781. doi:10.3168/jds.2016-12394

Sobczuk-Szul, M., Z. Wielgosz-Groth, M. Wroski, M. and A. Rzemieniewski. 2013. Changes in the bioactive protein concentrations in the bovine colostrum of Jersey and Polish Holstein - Friesian cows. Turk. J. Vet. Anim. Sci. 37:43-49.

Suárez-Mena, F.X., W. Hu, T.S. Dennis, T.M. Hill, and R.L. Schlotterbeck. 2017. $\beta$-Hydroxybutyrate (BHB) and glucose concentrations in the blood of dairy calves as influenced by age, vaccination stress, weaning, and starter intake including evaluation of BHB and glucose markers of starter intake. J. Dairy Sci. 100:2614-2624. doi:10.3168/jds.2016-12181

Svensson, C., and P. Liberg. 2006. The effect of group size on health and growth rate of Swedish dairy calves housed in pens with automatic milk-feeders. Prev. Vet. Med. 73:43-53. doi:10.1016/j.prevetmed.2005.08.021

Svensson, C., K. Lundborg, U. Emanuelson, and S.O. Olsson. 2003. Morbidity in Swedish dairy calves from birth to 90 days of age and individual calf-level risk factors for infectious diseases. Prev. Vet. Med. 58:179-197. doi:10.1016/S01675877(03)00046-1

Tedeschi, L.O., and D.G. Fox. 2009. Predicting milk and forage intake of nursing calves. J. Anim. Sci. 87:3380-3391. doi: $10.2527 /$ jas.2009-2014

Terré, M., E. Pedrals, A. Dalmau, and A. Bach. 2013. What do preweaned and weaned calves need in the diet: A high fiber content or a forage source? J. Dairy Sci. 96:5217-5225. doi:10.3168/jds.2012-6304

Terui, H., J.L. Morrill, and J.J. Higgins. 1996. Evaluation of wheat gluten in milk replacers and calf starters. J. Dairy Sci. 79:1261-1266. doi:10.3168/jds.S0022-0302(96)76480-9

Thiha, A., and F. Ibrahim. 2015. A colorimetric Enzyme-Linked Immunosorbent Assay (ELISA) detection platform for a pointof-care dengue detection system on a lab-on-compact-disc. Sensors (Basel) 15:11431-11441. doi:10.3390/s150511431

Thu-Hang, B.P., J. Dicksved, K.S. Sjaunja, and E. Wredle. 2017. Colostrum quality, IgG absorption and daily weight gain of calves in small-scale dairy production systems in Southern Vietnam. Trop. Anim. Health Prod. 49:1143-1147. doi:10.1007/s11250-017-1308-6

USDA. 2007. Dairy 2007: Heifer calf health and management practices on U.S. dairy operations, 2007. USDA, USA.

USDA. 2013. Carne de ternero la granja a la mesa. USDA, USA. https:/www.fsis.usda.gov/wps/portal/informational/enespanol/hojasinformativas/preparacion-de-las-carnes/carne-de-ternero/carne-de-ternero-granja-a-la-mesa (consultado 13 may. 2020).

Zarei, S., G. Reza Ghorbani, M. Khorvash, O. Martin, A. Hossein-Mahdavi, and A. Riasi. 2017. The impact of season, parity, and volume of colostrum on Holstein dairy cows colostrum composition. Agric. Sci. 8:572-581. doi:10.4236/as.2017.87043 